\title{
NOISE RADAR OF MILLIMETER RANGE
}

\section{Vladimir V. Kolesov, Evgeny A. Myasin}

Kotelnikov Institute of Radioengineering and Electronics of Russian Academy of Sciences, http://cplire.ru Moscow 125009, Russian Federation

kvv@cplire.ru, eam168@ms.ire.rssi.ru

Abstract. Practically all modern users of radar systems require an increase in the number and quality of information obtained from the observed space. Multifunctionality and efficiency of modern radar systems can be provided by the development and application of effective broadband technologies, unconventional digital algorithms and new adaptive applied solutions for the problem of processing signals and images in order to identify and recognize various classes of low-contrast objects. In the work on the basis of the IMPATT noise generator, a noise radar model working in the 8-mm range and having a frequency spectrum of the noise signal frequency up to $1 \mathrm{GHz}$ was developed and investigated. The layout of the model includes an IMPATT noise generator module based on a one-diode circuit operating in an 8-millimeter wavelength range with an integrated output power of $40 \mathrm{~mW}$ and a non-uniform spectral characteristic of about $6 \mathrm{~dB}$. Receiving and transmitting modules include antennas, waveguide elements: matching elements, impedance matcher, directional couplers, detector heads and attenuators. The computerized control unit provided program-algorithmic support of operating modes and double spectral processing of the signal. An experimental study of the noise radar model in the laboratory demonstrated a high resolution over a range of $15 \mathrm{~cm}$ (with an effective bandwidth of 800-900 MHz). The noise immunity of a radio engineering channel model based on a spread-spectrum signal was experimentally investigated. The maximum noise immunity for a radio channel with spreading was determined by the signalto-noise ratio at the receiver input, at which signal restoration becomes impossible for a given averaging time. In the noise immunity experiment, two types of interference were used: sinusoidal interference, close in frequency to the transmitted signal, and broadband interference matched to the transmitted signal by the spectrum. The results of experiments showed that for both types of interference, the maximum noise immunity is $\sim 25 \mathrm{~dB}$. Thus, ultra-wideband radar technologies based on noise signals are characterized by good electromagnetic compatibility, can be effectively used in the compilation of a radar portrait of an object, as well as in monitoring, positioning and control systems operating under conditions of intense interference.

Keywords: ultra-wideband technologies, complex signals, noise signals, radar, noise generator, millimeter range, dual spectral processing, noise immunity, resolution, electromagnetic compatibility

\section{UDC 621.391}

Bibliography - 27 references

\section{Content}

1. Introduction (235)

2. Methods for processing radar signals (237)

3. Structure of noise radar (238)

4. Measurement of range in noise radar (238)

5. Resolution and accuracy of spectral measurements (240)

6. Broadband diode generators of millimeter wave noise (242)

7. The model of the receiving and transmitting unit SSR (243)
8. Radar Distance Measurement (244)

9. Study of the resolving power of the SIR model for range (244)

10. Estimation of noise immunity of SHRL under the influence of narrowband and broadband interference (247)

11. Conclusion (248)

References (249)

APPENDIX

Myasin E.A. Noiseotron or my life in the 16th department of IRE RAS (250) 


\section{INTRODUCTION}

At present, a promising direction is actively developing in radar, representing broadband and ultra-wideband technologies. In the framework of this direction it is possible to move to a qualitatively new level of solving radar problems for remote detection of objects. In addition to the energy criterion (at the "yes" level "no") of detecting an object against the background of noise and the underlying surface, it is possible to proceed to the formation of a radar portrait of the object and to develop systems for automatic recognition of an object by its portrait, which qualitatively increases the information capabilities of radar systems (radar systems) . Radar with signals with a wide range of frequencies makes it possible to perform highly accurate, informative measurements of the parameters of reflecting objects under difficult electromagnetic conditions under the influence of active and passive interference $[1,2]$.

The main modern technologies that can ensure the realization of these opportunities are:

- technology of generation, emission and reception of broadband signals based on dynamic chaos [3],

- technologies for processing broadband signals based on fractal analysis and revealing the structural features of the target's radar portrait, which allows detecting and recognizing lowcontrast targets in real time in automatic mode when comparing the signal with a reference radar portrait [4].

One of the promising areas that significantly increase the information content of the radar is the use of short-pulse ultra-wideband signals with a spectrum width of $1 \mathrm{GHz}$ or more. In radar with UWB, the increase in informativeness is due to the reduction in the pulse volume of the radar by range. Thus, with a change in the duration of the probe pulse from $1 \mu \mathrm{s}$ to $1 \mathrm{~ns}$, the depth of the pulsed volume decreases from $300 \mathrm{~m}$ to $30 \mathrm{~cm}$. Ultra-wideband radars with pulsed radiation of less than one nanosecond duration have a high spatial resolution of the order of several centimeters when measuring range. When objects are detected at a significant distance from the radar, the necessary signal-to-interference ratio at the receiver input is achieved due to the giant peak power for single ultrashort pulses $[5,6]$.

In radars with continuous in time radiation of ultrabroadband sounding signals, for example, noise signals, it is possible to obtain the same signal-to-noise ratio at the input of the receiver when the average power of continuous radiation is reduced many-fold. The noise signals in the radar receiver are compressed in the time domain due to the correlation convolution, or in the frequency domain during the double spectral processing [7].

Noise UWB radars continuously emit random electromagnetic signals with a low spectral power density into the surrounding space. Along with the high informativeness and resolution of the measurements, noise radars are characterized by the secrecy, low probability of interception (Low Probability of Intercept) of their own noise emissions and electromagnetic compatibility with other operating means, including narrow-band systems [8].

A certain disadvantage for all radars with continuous radiation is the penetration into the receiving path of the signal from the transmitter, therefore the problem of forming a narrow radiation pattern and efficient shielding of the antennas seems to be quite important [9].

The increase in the accuracy and resolution of radar measurements is due to the complication of the structure and the expansion of the frequency band of the sounding signal. The main requirements for noise radars are high information content and resolution of measurement, stealth, low probability of interception of intrinsic noise emissions and electromagnetic compatibility with other operating facilities, including narrowband systems [10].

The use of complex broadband and ultrabroadband probing signals is one of the promising directions in radiolocation. The use of signals generated on the basis of chaotic algorithms allows to achieve practically the highest noise immunity of the radar to the effect of various interference [11]. The development of the technology of discrete systems and digital processing practically at the frequencies of sounding signals significantly expands the boundaries of the use of 
modern radar systems based on phased arrays with adaptive control of their modes and joint processing of signals.

The use of UWB signals in the radar allows:

- to improve the accuracy of measuring the distance to the object and the resolution of the range and angular coordinates, the effectiveness, stability of the radar to the effects of external and narrowband electromagnetic radiation, and interference;

- recognize classes and types of objects;

- simplify the protection equipment against all kinds of passive interference; to eliminate interference dips in the radiation pattern when observing the object at low angles.

A promising application of broadband signals in radar is the use of phase-manipulated signals of continuous radiation with a large base (up to 106) and a minimum peak power [12]. Such SH signals are called noise-like probing signals. Radar systems with a SH signal of detection of ground objects have increased noise immunity and stealth operation [13].

The most complete requirements for simultaneous resolution in range and speed are the noise signals. The main advantages of noise signals in comparison with other complex signals are the following:

- the noise sounding signal is similar to the internal noise of the receiver of the radio technical reconnaissance station, which provides a significant increase in the stealth operation of the radar, which makes it difficult, first, to register the fact of the radar operation and, secondly, to determine the parameters of its probing signal;

- taking into account the prospects of translating the short-range radar into a millimeter range of radio waves, it should be borne in mind that a radar with a noise-like signal can be simply coupled with passive location systems;

- from the general provisions of information theory it follows that the complication of the waveform and the increase in its duration makes it possible to increase its informativeness [14].

\section{METHODS FOR PROCESSING RADAR SIGNALS}

Signal processing methods are an integral part of radar information acquisition (RLI), the mathematical description of which is rather complicated. The processing procedure for radar signals is one of the most important processes of highlighting useful information. A special place is also given to equipment that can be successfully used to solve problems of constructing radio images from the data of reflected signals from detected objects [15].

In radars with continuous in time radiation of ultrabroadband sounding signals, for example noise signals, it is possible to obtain the same signal-to-noise ratio at the receiver input when the average power of continuous emissions is reduced many-fold. Compression of noise signals in the radar receiver is performed in the time domain due to correlation convolution, or in the frequency domain in the process of double spectral processing. Radar with dual spectral processing is based on the interference of completely incoherent reference and received noise signals $[16,17]$. The application of methods for spectral processing of the noise signal makes it possible to improve the accuracy of radar measurements, since frequency measurements currently have the highest possible accuracy.

Double spectral processing, sometimes referred to as cepstrum processing, is an alternative method to the correlation processing of signals in noise radar. As a result of double spectral processing, it is possible to obtain the response component at the output of the receiver as a function of the mutual correlation of the emitted signal and noise reflections from the detected object. The effect of interference with the distributed spectrum on the receiver leads to a decrease in the correlation peak in the secondary spectrum relative to the noise level [19].

\section{STRUCTURE OF NOISE RADAR}

The radar noise signal $n(t)$ with frequency band $\Delta \mathrm{f}$ is assumed to be stationary and ergodic, which allows us to find the statistical characteristics of random processes, averaging in time individual implementations. This circumstance is important 
in noise radar in the case of correlation or double spectral processing of continuous noise signals in real time. The procedure of averaging over an ensemble of a set of statistically independent realizations of random processes is a convenient method of theoretical investigation in noise radar, however, it is rarely used in practice when measuring the mutually correlated function of the emitted and received noise signals due to the complexity or impossibility of realizing the averaging procedure in real time.

At the output of the noise generator, part of the radiated signal branches into the receiver channel of the receiver. The level of the reference signal can be smoothly controlled by means of a controlled attenuator. The reference signal is fed to the input of the linear adder in the radar receiver circuit. On the other input of the linear adder comes an additive mixture of signals from the receiving antenna. The fundamental difference between radar based on interference and double spectral processing of broadband signals is the operation of linear summation of the received sounding and reference noise signals in the linear part of the receiver.

Dual spectral processing of radar signals is performed in the frequency domain, in contrast to the correlation convolution of signals, which occurs in the time domain. Alternative methods of radar with correlation and double spectral processing of signals have fundamental differences in receiver design schemes, however, lead to close results in the implementation of radar measurements [20].

A typical form of the model spectrum of the total noise signal is shown in Fig. 1 fragment of the spectrogram at the mean frequency $f_{0}=3050$ MHz. The spectrum fragment in the $100 \mathrm{MHz}$ band is an interference pattern of 10 alternating

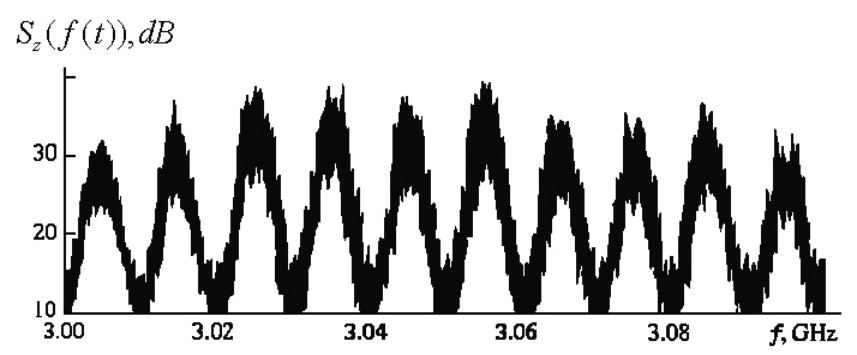

Fig. 1. Fragment of the spectrum of the ultra-wideband total noise signal. bands for the power spectral density, depending on the current frequency $f$.

The initial spectrum of the total noise signal extends over a wide frequency band $\Delta f \approx 2000$ $\mathrm{MHz}$ with a lower frequency $f_{1} \approx 2000 \mathrm{MHz}$ and an upper frequency $f_{\mathrm{h}} \approx 4000 \mathrm{MHz}$. The fragment of the spectrum in Fig. 1 is measured by probing a point object remote from the locator at a distance $L=15$ meters. The signal reflected from the object is delayed for a time $T=2 \mathrm{~L} / \mathrm{c}=10^{-7}$ seconds. The number of interference fringes or maxima in the initial spectrum of the total signal will amount to a large value $N=\Delta f T=200$.

When the frequency band $\Delta f$ of the sounding noise signal decreases, the number of interference maxima and minima in the spectrum of the total signal decreases. When the frequency band is $\Delta f=10 \mathrm{MHz}$, only one interference maximum $N=\Delta f T=1$ is observed in the spectrum. The interference of partially coherent noise signals with a narrow frequency band $\Delta f<10 \mathrm{MHz}$ occurs under the condition $T \leq \tau_{c}$ when the relative delay $T=10^{-7} \mathrm{~s}$ becomes less than the coherence time $\tau_{\mathrm{c}} \approx 1 / \Delta f$.

\section{MEASUREMENT OF RANGE IN NOISE RADAR}

When adding incoherent delayed signals, a periodic interference pattern is observed in the spectral range of the measurements. The spectrum of the total signal is modulated by a function with a period $F_{m}=1 / T$ inversely proportional to the relative delay of the signals.

The period of modulation of the $F_{m}$ spectrum in the frequency domain is inversely proportional to the relative delay $T$ of the probing signals:

$$
F_{m}=\frac{1}{T}=\frac{1}{\left|T_{1}-T_{0}\right|} \text {. }
$$

Taking into account the relation $T_{1}=2 L_{1} / c$, it follows from expression (1) that the required range to the target is uniquely calculated as:

$$
L_{1}=\frac{c}{2}\left[T_{0}+\frac{1}{F_{m}}\right] \text {. }
$$

Here, $T_{0}$ is a known path delay in the reference channel.

The incoherence condition for the delayed signals is written in the form 


$$
\Delta f / F_{\mathrm{m}}>>1
$$

It follows from (3) that when interference of completely incoherent noise signals is interfered, many $F_{m}$ scales of periodic modulation of the spectrum (9) in the frequency band $\Delta f$ of the radar signal are stacked.

In the spectrum band $\Delta f$ of the total signal, there are many interference fringes (Fig. 1) and many $F_{\mathrm{m}}$ scales of periodic modulation are stacked so that $\Delta f / F_{\mathrm{m}}>1$. The period $F_{\mathrm{m}}=$ $1 / T$ of the spectral modulation contains useful information about the relative delay $T=T_{1}-T_{0}$ of the radar signals. If we measure the period $F_{m}$ of spectral modulation, we can uniquely determine the required range by formula (2).

The secondary spectrum $G_{s}(F)$ contains a useful spectral peak at the middle frequency $F_{1}$ and low-frequency components of the signal and interference concentrated mainly near the zero frequency (Fig. 2).

The appearance of an information peak in the secondary spectrum $G_{\mathrm{s}}(F)$ as a function of crosscorrelation between the reflected and reference signals leads to the detection of a remote object by a radar with double spectral processing of the ultrawideband noise signal.

During the calculation of the secondary spectrum $G_{\mathrm{s}}(\mathbf{F})$, an information spectral peak is extracted and a remote object is detected. To determine the parameters of the object, the average frequency $F_{1}$ is measured, as well as the information peak level. The required range to the object is uniquely determined by the formula

$$
L_{1}=\frac{c}{2}\left[T_{0}+\frac{F_{1}}{V_{s}}\right] \text {. }
$$

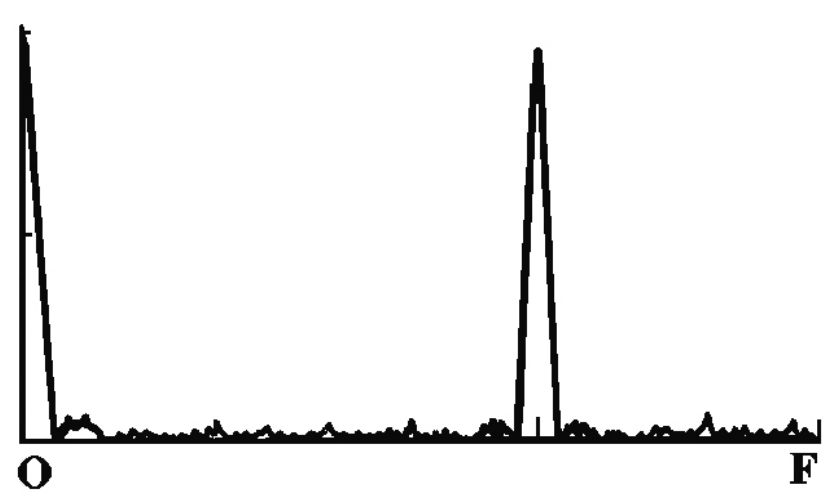

Fig. 2. Secondary spectrum of the total noise signal.
Thus, the unambiguous determination of the target range $L_{1}$ and the transmission coefficient $\mathrm{h}$ in the propagation channel is realized as a result of the second Fourier transform for the spectrum of the sum signal.

The level of low-frequency components can exceed the information peak under the influence of strong interference. To avoid distortion of the information peak, it is possible to compensate for interfering low-frequency components in the secondary spectrum. For this purpose, a double spectral analysis of the received radiation is performed in the absence of useful reflections, when there is no information peak in the secondary spectrum. As a result of the mathematical operation of subtraction of secondary spectra in the presence and absence of a useful signal, only the information peak remains in the form of the envelope of the cross-correlation function. The average frequency $F_{1}$ of the information peak uniquely determines the required range to the object.

A radar receiver with dual spectral analysis performs a measurement of the cross-correlation function for the reflected and emitted signals. Such a receiver can be considered as an optimal receiver of the correlation type in the first approximation for a long averaging time, when the variance of the random estimate for the measured cross-correlation function is small [21].

\section{RESOLUTION AND ACCURACY OF SPECTRAL MEASUREMENTS}

The resolving power of radar measurements is determined by the possibility of distinguishing two closely located objects. The marks from two objects with delays $T$ and $(T+\Delta T)$ can be resolved by measuring the secondary spectrum if the corresponding informative spectral peaks $G_{2}(F-F T)$ and $G_{a}(F-F T+\Delta \mathrm{T})$ are frequency-separated by the width of the spectral peak (рис. 3).

Each spectral peak in the secondary spectrum is described by a cross-correlation function for signals with a corresponding relative delay. The width of each spectral peak is $\Delta F_{c}=V_{s} \cdot \tau_{c}$, and is determined by the coherence time $\tau$ c of the radar signal to within a constant $V_{\mathrm{S}}$. 
Thus, two spectral peaks in the secondary spectrum are resolved if they are shifted by the frequency $\Delta F=F T+\Delta T-F T=V_{s} \Delta T$, which exceeds the width of the spectral peak $\Delta F_{\mathrm{c}}=V_{\mathrm{s}} \tau_{\mathrm{c}}$ $\Delta F \geq \Delta F_{c}$ or $\Delta T \geq \tau_{c}$.

The range resolution for the location of two point reflectors located at a distance $L$ and $L+\Delta L$ amounts to

$$
\Delta L=\frac{c}{2 \Delta f} \text {. }
$$

Each informative peak $G_{a}(F-F T)$ and $G_{a}(F-F T+$ $\Delta T)$ in the secondary spectrum is a cross-correlation function for the reference signal reflected from the given target. When probing targets with noise signals with a uniform spectrum in the frequency band $\Delta f$, a cross-correlation function is measured, the width of the main lobe at the level of the first minimum is $\tau=1 / \Delta f$ and inversely proportional to the band of the probing signal. In the case of double spectral processing, two reflected signals differ if they are separated in time by an interval that exceeds the correlation time.

An estimate of the accuracy of measuring the range to the object $L$ is found by differentiating expression (6)

$$
\delta L=\frac{c}{2}\left[\delta T_{0}+\frac{F}{V_{s}} \frac{\delta F}{F}-\frac{F}{V_{s}} \frac{\delta V_{s}}{V_{s}}\right] .
$$

The differential (7) reduces to the form

$$
\delta L=\left[L-\frac{c T_{0}}{2}\right]\left[\frac{\delta F}{F}-\frac{\delta V_{s}}{V_{s}}\right]+\frac{c \delta T_{0}}{2} .
$$

Here $\delta F / F$ is the relative error of the measurement of the mean frequency $F$ for the information peak in the secondary spectrum $G_{\mathrm{S}}(F)$. The differential (8) also depends on the relative error $\left(\delta V_{\mathrm{s}}\right) / V_{\mathrm{S}}$ of determining the $V_{\mathrm{s}}$ frequency

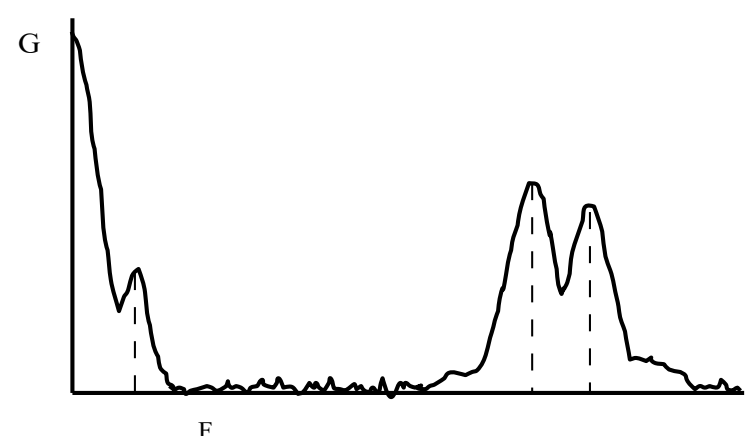

Fig. 3. Spectral resolution of two point reflectors. for the first spectrum analyzer and on the error in determining the constant delay $\delta T_{0}$ in the receiver reference channel. In turn, the secondary spectrum of $G_{S}(F)$ is calculated by Fourier transform from the primary spectrum. The relative error $\delta F / F$ of the measurement of the mean frequency $F$ for the information peak in the secondary spectrum $G_{\mathrm{S}}(F)$ is determined by the relative error $\delta f / \Delta f$ of the measurement of the primary spectrum with the frequency band $\Delta f$. Then the expression for the square of the error in measuring the range $L$ is reduced to the final form.

Here $\delta F / F$ is the relative error of the measurement of the mean frequency $F$ for the information peak in the secondary spectrum $G_{\mathrm{S}}(F)$. The differential (8) also depends on the relative error $\left(\delta V_{\mathrm{s}}\right) / V_{\mathrm{s}}$ of determining the $V_{\mathrm{s}}$ frequency for the first spectrum analyzer and on the error in determining the constant delay $\delta \mathbf{T}_{0}$ in the receiver reference channel. In turn, the secondary spectrum of $G_{\mathrm{S}}(F)$ is calculated by Fourier transform from the primary spectrum. The relative error $\delta \mathbf{F} / \mathbf{F}$ of the measurement of the mean frequency $F$ for the information peak in the secondary spectrum $G_{\mathrm{S}}(F)$ is determined by the relative error $\delta f / \Delta f$ of the measurement of the primary spectrum with the frequency band $\Delta f$. Then the expression for the square of the error in measuring the range $L$ is reduced to the final form

$$
(\delta L)^{2}=\left(L-\frac{c T_{0}}{2}\right)^{2}\left[\left(\frac{\delta f}{\Delta f}\right)^{2}+\left(\frac{\delta V_{s}}{V_{s}}\right)^{2}\right]+\left(\frac{c \delta T_{0}}{2}\right)^{2} .
$$

The error in measuring the range becomes smaller with decreasing errors $\left(\delta V_{\mathrm{s}}\right) / \mathrm{V}_{\mathrm{S}}$ and $\delta T_{0}$. If there is no error when setting the frequency scan rate $\delta V_{\mathrm{s}}=0$ and the constant delay in the reference channel is known to be $\delta T_{0}=0$, then the error in measuring the range to target $L$ is in the form

$$
\delta L=\left[L-\frac{c T_{0}}{2}\right] \frac{\delta f}{\Delta f} .
$$

Those. the distance measurement error decreases with increasing the frequency band of the probing signal $\Delta f$ and with the improvement of the resolving power $\delta f$ of the first spectrum analyzer [22].

Thus, the main results of applying double spectral processing of the signal are as follows.

When the fully incoherent reflected from the object and the reference signal are added, a periodic 
interference pattern is observed in the spectral range of the measurements. The spectrum of the total signal is modulated by a function with a period inversely proportional to the relative delay of the signals. In the spectrum of the total signal, there are many interference fringes and many scales of periodic modulation are laid down, when the relative delay of the signals is many times greater than the coherence time.

Measurement of the period of spectral modulation is due to the Fourier transform of the spectral density of the total signal as a periodic function of frequency. The secondary spectrum of the total signal contains a useful spectral peak at the middle frequency and low-frequency components near the zero frequency. A useful spectral peak corresponds to a mutually correlated function for the delayed and reference radar signals. The average frequency of the information peak is determined by the relative delay of the signals, and the magnitude of the peak depends on the attenuation of the signals in the propagation channels. Measuring the frequency and level of the spectral information peak, uniquely determine the distance to the object and the transmission coefficient in the propagation channel.

The resolving power and accuracy of radar measurements by the method of double spectral analysis are determined by the frequency band of the sounding signal. The accuracy of the range measurement is enhanced by improving the resolving power of the first spectrum analyzer and by introducing a known delay in the radar reference channel.

Dual spectral analysis is a quasi-optimal signal processing in the sense that the secondary spectrum of the sum signal contains informative peaks as a cross-correlation function for useful reflections and a reference signal.

In addition to informative peaks at the main frequencies, the secondary spectrum contains combinational components at the difference frequencies. Each combination component at the difference frequency is a cross-correlation function for a pair of signals received from the respective partial reflectors. The appearance of combinational components at the difference frequencies in the secondary spectrum indicates the detection of a complex object from partial point reflectors. The largest frequency in the spectrum of the combination components determines the radial size of the complex object.

The appearance of combinational components at the difference frequencies may cause difficulties in identifying the main spectral peaks in the secondary spectrum. To avoid ambiguity of the range measurements, it is necessary to introduce additional delay into the receiving channel and to carry out the frequency separation of the spectral peaks in the secondary spectrum. Then the main spectral peaks are shifted to the high-frequency part of the secondary spectrum, and the combination peaks remain in the low-frequency region. The boundary frequency separating the main and combination peaks in the secondary spectrum is determined by the largest radial dimensions of the complex object.

The detection zone for a radar with spectral analysis can extend directly from the transceiver antennas if an additional delay is set in the receiving path for the required value and the condition for interference of completely incoherent signals is satisfied. The greatest detection range is determined by the resolution of spectral analysis by frequency. The increase in the maximum detection range is achieved by a constant delay in the radar reference channel.

The spectral method of radar makes it possible to obtain the same information about the range as in the case of a mutually correlated method, but it has no practical limitations when using ultra-wideband noise signals. With the increase in the frequency band of the probing signals to units of gigahertz, the implementation of noise correlators with controlled or multi-lead delay lines for a priori unknown time is a technically difficult task.

Information about the range to the object is continuously distributed over the entire spectrum of the ultra-wideband total signal. The true range can be determined from fragments of the general spectrum, when other parts of the spectrum are lost or struck, for example, by strong active interference. In this case, the resolving power decreases, and the informativeness of spectral interferometry. A similar effect of restoring information on the part of the 
interference pattern is observed in coherent optics and holography.

\section{WIDEBAND MILLIMETER WAVE DIODE NOISE GENERATORS}

The principles of stochastization of oscillations in electron-wave systems with delay were used as the basis for constructing experimental models of diode GSh. A comparative analysis of the characteristics of the noise signal of solidstate semiconductor generators using Gunn diodes and avalanche-passing diodes (PDDs) in the centimeter wavelength range determined the choice of the active element for the noise generator in favor of the latter. LDPs were also used in noise generators (GSLPDs) of the millimeter wave band [23].

Low-power generators (with a power of up to $100 \mathrm{~mW}$ ) can be developed in a solid-state version using a single-diode circuit using an LAP. The analysis of the domestic element base showed that domestic industrial single-pass diodes $2 \mathrm{~A} 717 \mathrm{~A}-\mathrm{G}$, $33 \mathrm{GHz}-37.5 \mathrm{GHz}$ range, or $\mathrm{KA} 717-\mathrm{V}, \mathrm{G}$ range $37.5 \mathrm{GHz}-53.5 \mathrm{GHz}$ can be used as the LAP, which provided in the single-mode generators of harmonic oscillations the output power up to 100 $\mathrm{mW}$ and overlapped the range from $8 \mathrm{~mm}$ to $6 \mathrm{~mm}$. In this case, the range of operating currents of these diodes ranged from $100 \mathrm{~mA}$ to $150 \mathrm{~mA}$, and the breakdown voltage was $20 \ldots 22 \mathrm{~V}$. For working with diodes of the KA-717-A-G series, a special power source was created, which ensures the operation of the LAP at high breakdown voltages and large operating currents.

The waveguide design of a single-mode harmonic oscillator on the LPD (GLPD) with an open-cavity open-cavity resonator was taken as the basis of the generator design. Schematically, the design of the resonator chamber of the GLDD is shown in Fig. 4.

In Fig. 4 that the autogenerator includes the following elements: an open-wave open resonator formed by a disk terminating the current lead and a heat sink of the LAP, a resonance frequency tuning device (movable plunger) and a decoupling filter in the power circuit and an additional tunable resonator (in Fig. 4, not shown). The adjustment of the communication with the load was carried out with a coupling screw located at the center of the wide wall of the waveguide between the diode and the load. The diode was placed in a standard waveguide $7.2 \times 3.4 \mathrm{~mm}$. No other filtering devices such as resonant windows or resonance gaps have been used

In this design, by regulating the current through the diode and tuning elements, it is possible to obtain in stellar oscillation mode a stochastic oscillation mode with a bandwidth of $\sim 400 \mathrm{MHz}-000 \mathrm{MHz}$ and an output power of $\sim 50 \mathrm{~mW}$... $30 \mathrm{~mW}$, respectively, in the $8 \mathrm{~mm}$ wavelength range.

The phenomenon of stochastization of oscillations is a fundamental property of a nonlinear autooscillatory dynamical system, and the state of generation of stochastic (noise) oscillations is as natural as generation of regular oscillations. The oscillatory system GSLPD does not have an external feedback line, the change in the transmission coefficient of which can change the degree of nonlinearity in the system. It is a complex multimode resonator, and therefore it is impossible to single out one control parameter, the variation of which could change the operating modes of the generator without changing the nonlinear characteristic of its active element or the property of the oscillatory system [24]. This circumstance substantially complicates the search and adjustment of stochastic regimes, both in the experimental investigation of such a generator, and in the mathematical modeling of processes occurring in it [25]. Therefore, for the experimental

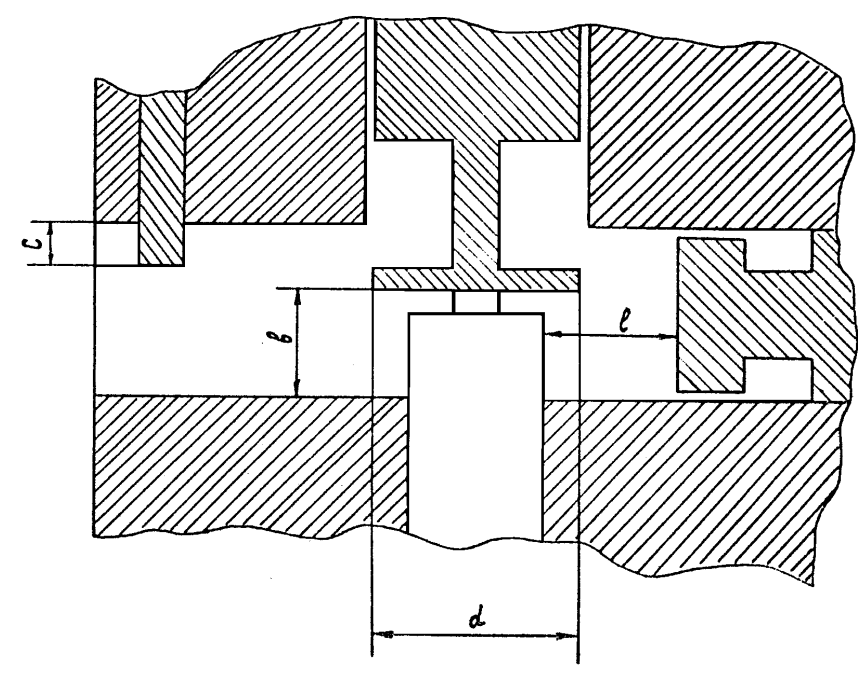

Fig. 4. Construction of a resonator chamber of a GLPD. 
investigation of the signal generated by the GLDD, depending on the variation of various control elements of the structure and current through the diode, a special stand was created, which allows measuring not only the output power, but also the spectral characteristic of the generated signal. It should be noted that for some applications it is necessary to have an idea of the distribution of the probabilities of the signal or of the distribution of the probabilities of its envelope, on the basis of which one can judge the degree of their proximity to the Gaussian or Rayleigh distribution, respectively. This fact has sufficiently served as a basis for choosing an LAP as an active element for a noise generator [26].

\section{MODEL OF THE RECEIVING AND TRANSMITTING UNIT SSR}

A block diagram of the layout of the receiving and transmitting block SSR is shown in Fig. 5. The measuring part of the noise radar provides a double spectral processing of the total signal to determine the range to the object and contains two spectrum analyzers (AS) in its composition. The first one is high-frequency, with the help of which the spectrum of the total signal (the signal reflected from the object + reference signal) is analyzed at the carrier frequency in the millimeter wavelength

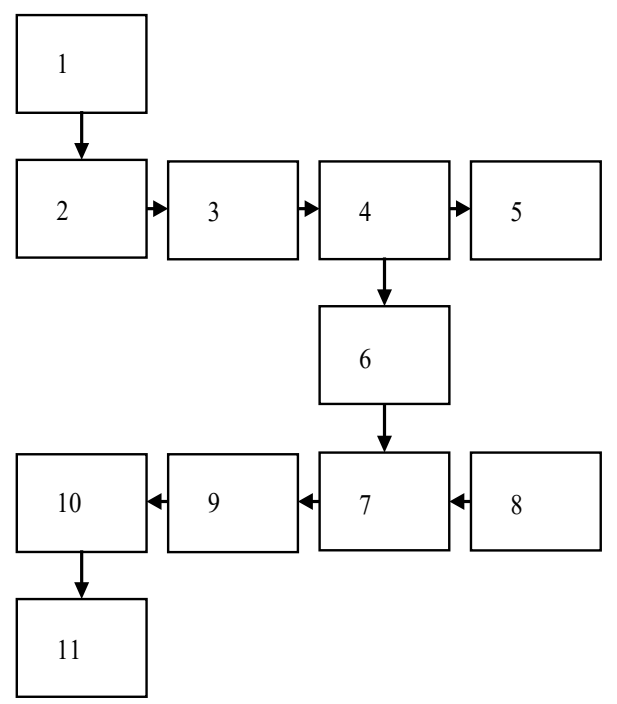

Fig. 5. Block diagram of the receiving and transmitting unit SSR: 1-power supply, 2 - noise generator $8 \mathrm{~mm}$ of the wave band, 3 - gate-isolation, 4 - directional coupler of the reference signal, 5 radiating antenna, 6 - attenuator, 7 - adder received and reference signals, 8 - receiving antenna, 9 - spectrum analyzer, 10 - ADC,

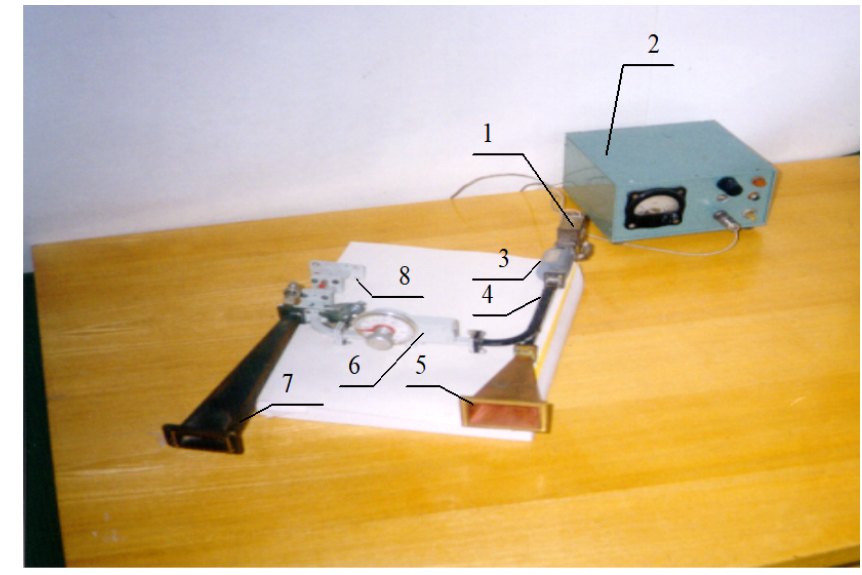

Fig. 6. Model of the receiving and transmitting unit SSR: 1 LDP-noise generator of $8 \mathrm{~mm}$ wave band, 2 - power supply, 3 ferrite isolation valve, 4 - directional coupler of the reference signal, 5 - emitting horn, 6 - attenuator, 7 - receiving horn, 8 - adder of the received and reference signals.

range. The second spectral transformation is carried out using a standard ADC connected to a personal computer.

The hardware part of the noise radar is shown in Fig. 6.

The width of the GS spectrum can be adjusted by means of the operating current through the diode. For example, at a diode current of $\mathrm{I}=93$ $\mathrm{mA}$, the spectral width is $700 \mathrm{MHz}$, and at a current $\mathrm{I}=100 \mathrm{~mA}$, the width of the GS spectrum is 800 $\mathrm{MHz}$. The output power is $3.6 \mathrm{~mW}$ and $4.4 \mathrm{~mW}$, respectively.

\section{RADAR MEASUREMENT OF DISTANCE}

To test the calculated theoretical relationships made in the analysis of the double spectral signal processing method, when measuring distances using the developed transceiver unit under laboratory conditions, test measurements were made at distances $\mathrm{L}_{1}=3.5 \mathrm{~m}$ and $\mathrm{L}_{2}=10 \mathrm{~m}$ to the signal reflector. Calibration of the SIR layout consists in determining the hardware constants that depend on the relative position of the reflecting antennae of the reflector required for metrological radar measurements. The mobile stand with the SCHL layout is shown in Fig. 7.

For the two measured distances, the corresponding coefficients $\alpha$ and $\mathrm{L}_{0}$ were found, which are constant for a given SSR configuration, as a result of solving the system of equations: 


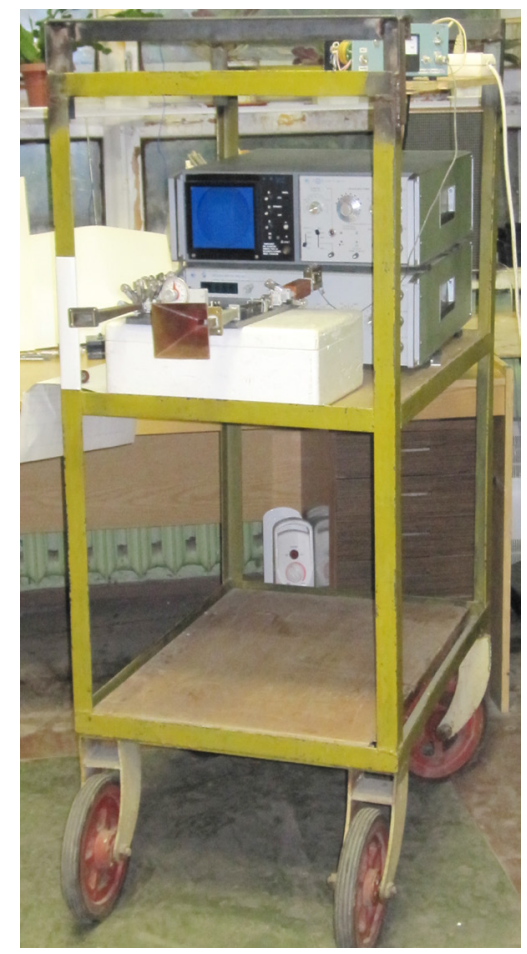

Fig. 7. Mobile stand with SCHL layout.

$$
\begin{aligned}
& L_{1}=\alpha \Omega_{1}+L_{0}, \\
& L_{2}=\alpha \Omega_{2}+L_{0},
\end{aligned}
$$

where $\Omega_{1}=99.4 \mathrm{~Hz}, \Omega_{2}=293.3 \mathrm{~Hz}$ are the measured frequencies in the secondary signal spectrum after double spectral processing.

As a result of solving the system of equations (11), the following values of these constants were obtained: $\alpha=0.0335, \mathrm{~L}_{0}=0.174 \mathrm{~m}$.

To check the coefficients obtained, measurements were taken at intermediate distances of $6.37 \mathrm{~m}$ and $7.1 \mathrm{~m}$ from the measured values of modulation frequencies $\Omega_{3}=185 \mathrm{~Hz}$ and $\Omega_{4}=207 \mathrm{~Hz}$, obtained by double spectral processing of the signal from the spectrum analyzer C4-60. The control measurements of the distance by the laser range finder yielded an almost exact coincidence (up to $10 \mathrm{~cm}$ ) with radar measurements obtained by the double spectral signal processing method.

The modulation frequencies corresponding to different distances to the reflector in the primary spectrum were determined on the basis of a secondary spectral transformation of the primary total spectrum of the reflected signal with the reference signal. A photograph of the total spectrum of the reflected signal with a reference signal on the screen C4-60 is shown in Fig. 8.

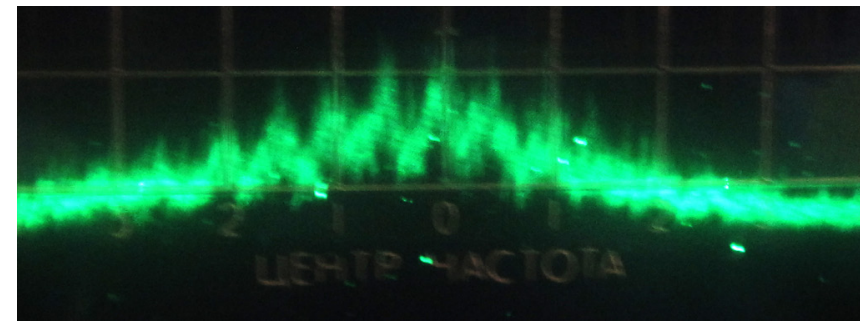

Fig. 8. The total spectrum of the reflected signal with the reference signal on the screen C4-60 (scanning scales -50 MHz / div).

\section{STUDY OF THE RESOLVING POWER OF THE SIR MODEL FOR THE RANGE}

To realize the maximum range resolution it was necessary to extend the band to the maximum possible in the given design of the transmitting module. For this purpose, a high-frequency diode KA717B was used in the GSh. In Fig. 9 shows the types of broadband spectra of the noise signal of the spectrum at different operating currents of the GSH. It should be noted that the expansion of the band reduces the integrated power of the noise signal to units of milliwatts.

As can be seen in Fig. 9, the maximum spectral width is $\approx 1000 \mathrm{MHz}$, while the high-frequency part of the spectrum (above $700 \mathrm{MHz}$ ) has a significant unevenness and a lower value of the noise power density.

To obtain the maximum amplitude in the modulation signal of the total frequency spectrum, it is necessary to vary the power level of the reference signal.

Adjustment of the tap-off power from the emitted noise signal was carried out using a calibrated precision attenuator. The calibration schedule of the attenuator is shown in Fig. 10.

In Fig. 11 is a photograph of the total spectrum of the reflected signal with a reference signal on the screen C4-60 at a distance to the reflector of $2.4 \mathrm{~m}$. Fig. 11 it can be seen that only the central part of the primary signal spectrum, shown in Fig. 8. Thus, the expected resolving power will be $\approx 0.14 \mathrm{~m}$.

In Figs. 12, 13 and 14 show the results of double spectral processing of the signal from the spectrum analyzer C4-60 at a distance of 2.4, 2.55 and $2.7 \mathrm{~m}$ from the reflector.

As seen in Fig.12, the mark from the reflector in the secondary spectrum is determined by the frequency $\Omega=143.3 \mathrm{~Hz}$. 


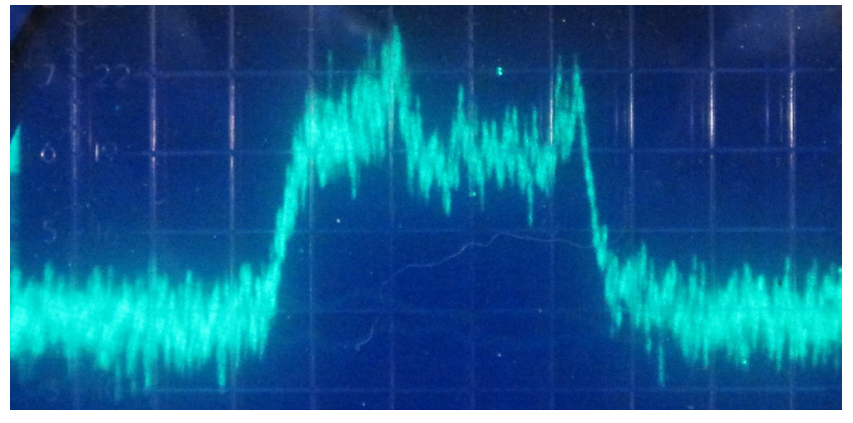

a) spectral characteristic of the GS in noise mode at a current of 136 $m A$. The scale is $200 \mathrm{MHz} /$ div. $P=4.3 \mathrm{~mW}$. The spectral width is $\sim 800 \mathrm{MH}_{2}$, the spectral unevenness is $\sim 3 \mathrm{~dB}$.

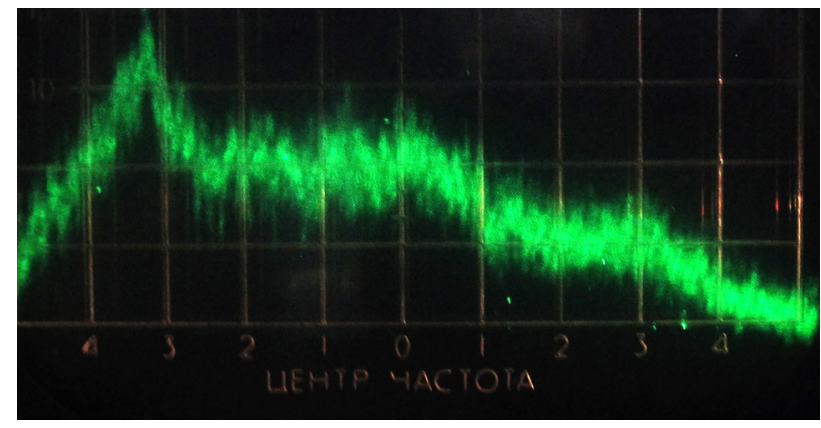

b-spectral characteristic of the GS in noise mode at a current of 145 mA. Scale $100 \mathrm{MHz}$ / div. $P=3.5 \mathrm{~mW}$. The spectrum width is $1000 \mathrm{MH}_{2}$, the spectral unevenness is $\sim 5 \mathrm{~dB}$.

Fig. 9. Types of spectra of the noise signal GSh.

As can be seen in Fig. 13, the mark from the reflector in the secondary spectrum is determined by the frequency $\Omega=150 \mathrm{~Hz}$.

From Fig. 14 that the mark from the reflector in the secondary spectrum is determined by the frequency $\Omega=156.55 \mathrm{~Hz}$.

Thus, the real resolving power of the SIR layout in determining the range is determined by the achieved characteristics of the noise signal and in this case is $15 \mathrm{~cm}$.

Obtaining the necessary radar characteristics by distance is provided by the use of existing transmitreceive modules of the appropriate power with the necessary input and output parameters. The values of all the basic parameters for the SSR can be obtained from the basic radar equation:

$$
R_{\max }=\sqrt[4]{\frac{P_{t r} G_{t r} G_{r c} S_{e f f} \lambda^{2}}{(4 \pi)^{3} P_{r c \text { min }}}},
$$

where $\mathrm{R}_{\max }$ is the maximum working range of the SSR, $\mathrm{P}_{\text {tr }}$ is the radiation power of the radio transmitter,

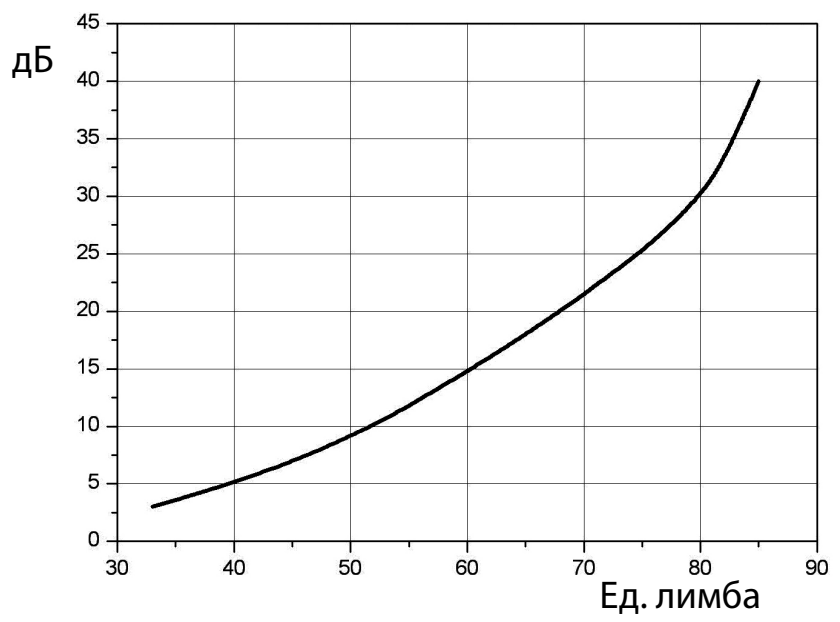

Fig. 10. The calibration schedule of the attenuator.
$P_{r c-m i n}$ is the minimum signal power at the receiver input, $G_{t r}$ and $G_{r c}$ are the transmission and receive antenna gain factors, $S$ is the effective scattering area of the reflector, and $\lambda$ is the wavelength of the emitted signal.

It follows from relation (12) that to increase the range it is necessary to increase the transmitter power, increase the sensitivity of the receiver, and use antennas with maximum amplification factors. Parabolic antennas in the MM-range of wavelengths have significant gain factors of $10^{4}-10^{5}$ depending on the dimensions of the paraboloid. Agilent Technologies is using exclusive technologies such as a low noise signal path and a special Noise Floor Extention technology to eliminate noise due to distortion, further expand the dynamic range and provide an average level of the receiver's own noise of $-140 \mathrm{dBm}$ at a frequency of $50 \mathrm{GHz}$

Thus, in order to provide an operational range of SHRL of the order of $500 \mathrm{~m}$ with an effective area of scattering of the object of $1 \mathrm{~m}^{2}$ with a potential resolution in the range of about $10 \mathrm{~cm}$ (1 $\mathrm{GHz}$ probing noise band), using $1 \mathrm{~m}$ parabolic antennas and a mixer sensitivity at the receiver input

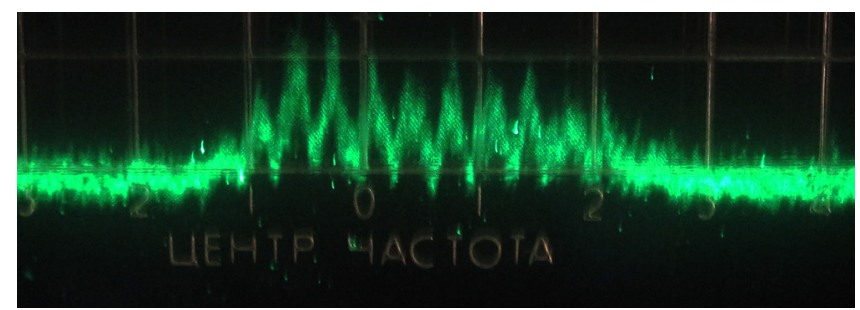

Fig. 11. The total spectrum of the reflected signal with the reference signal on the screen C4-60, scale -200 MHz / div (distance to the reflector $2.4 \mathrm{~m}$ ). 


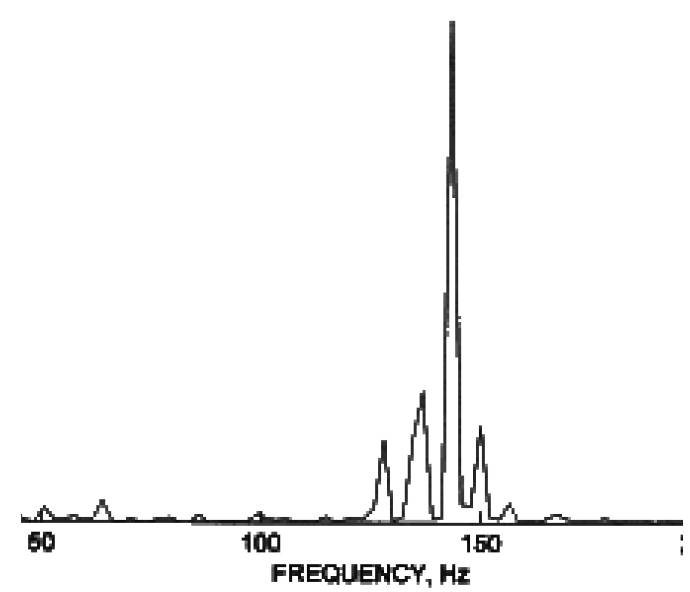

Fig. 12. The results of double spectral processing of the signal from the spectrum analyzer C4-60 at a distance to the reflector of $2.4 \mathrm{~m}$. $-140 \mathrm{dBm}$, it is necessary to use a transmitter with an integrated radiation power of the order of $200 \mathrm{~W}$.

\section{ESTIMATION OF NOISE IMMUNITY OF SHRL UNDER THE EFFECT OF NARROWBAND AND BROADBAND INTERFERENCE}

An important quality of noise radars with continuous emission is energy concealment from electronic reconnaissance means. The radiated signals of the SRLS at the input to the receivers are under noises and therefore are indistinguishable from them in terms of their correlation properties.

The extension of the signal bandwidth is usually achieved by such carrier wave modulation that forms a modulated signal with a bandwidth wider than the modulating function. An effective way of expanding the signal spectrum is its phase modulation [27].

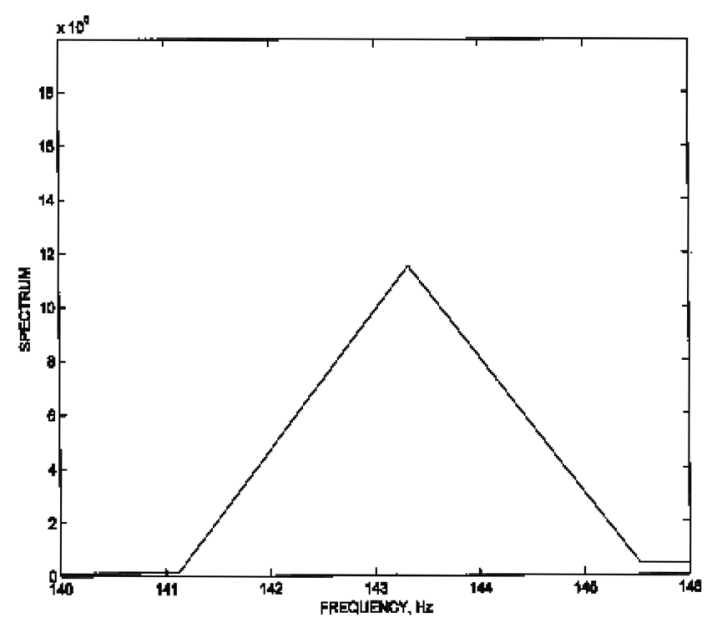

It is implemented on the basis of a discrete phase manipulator. In order to provide a wide frequency band, the use of a $\pi$-modulator is most effective, in which two states correspond to the absence of a phase shift (zero shift) and a phase shift by $\pi$. Such modulators are effectively implemented in bridge circuits with switching $\mathrm{p}-\mathrm{i}-\mathrm{n}$ diodes.

The noise immunity of a radio engineering channel model based on a noise-like carrier was experimentally investigated (Fig. 15). Coders implemented one of the developed algorithms for generating a class of chaotic signals.

The spread of the transmitted signal from the microwave generator (1) was extended by means of the microwave phase manipulator FM1 (3). The microwave signal at the middle frequency Fcp entered the FM1 input, whose control was carried out by a chaotic binary pulse train from the encoder (6). As a
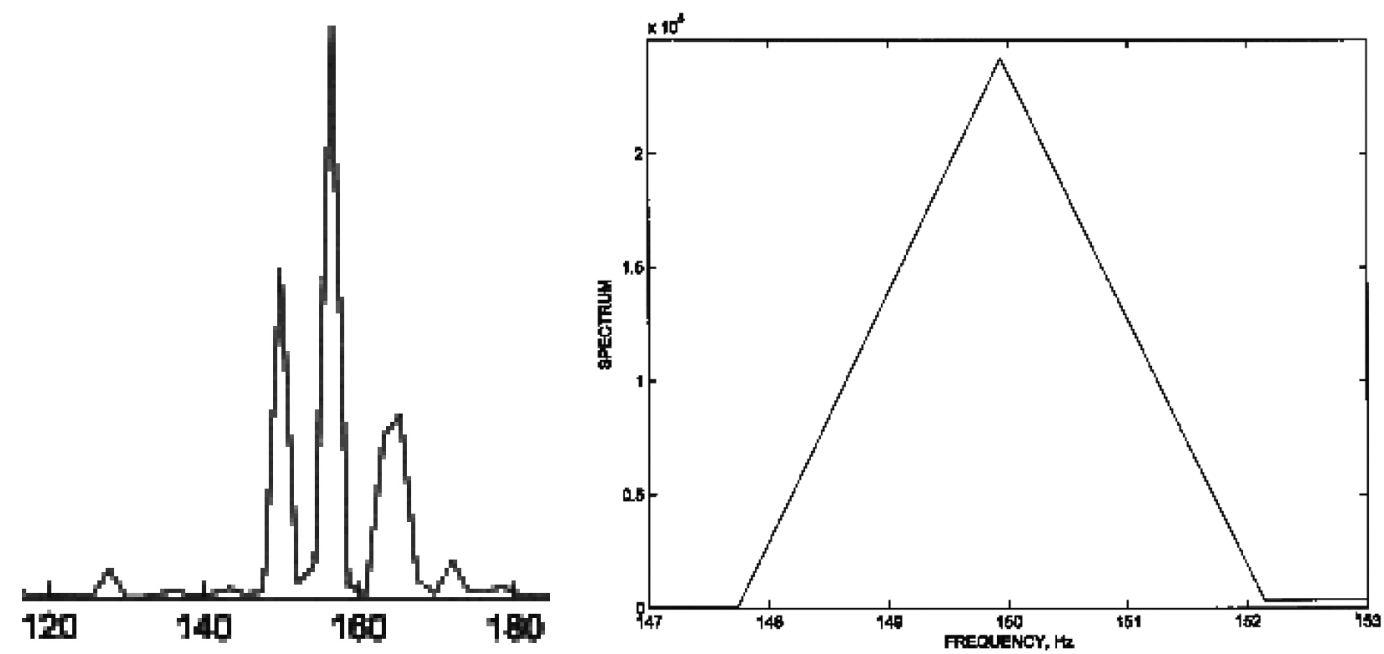

Fig. 13. Results of double spectral processing of the signal from the spectrum analyzer C4-60 at a distance to the reflector of $2.55 \mathrm{~m}$. 

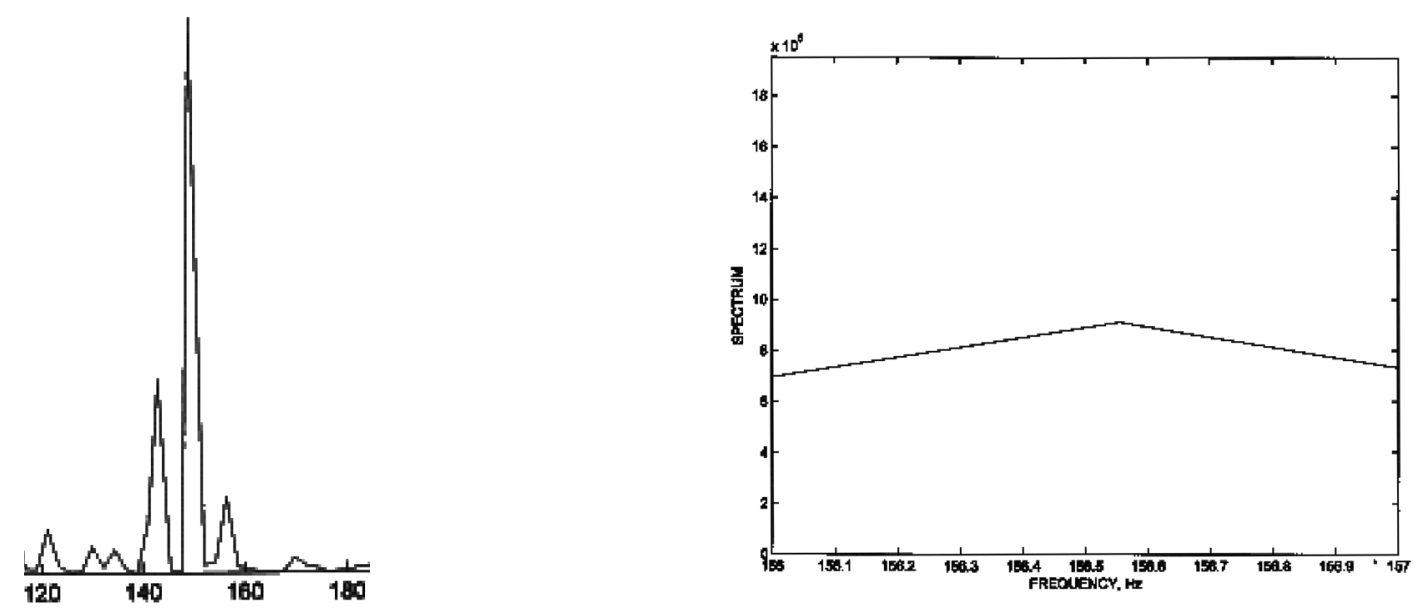

Fig. 14. The results of double spectral processing of the signal from the spectrum analyzer C4-60 at a distance to the reflector of $2.7 \mathrm{~m}$.

result, a noise signal with a continuous spectrum was observed at the output of FM1 (3).

The signal with the frequency Fm entered the input of a phase modulator controlled by a chaotic binary pulse train from the digital encoder. At the output, the signal had a continuous noiselike spectrum. To this signal the interference signal was mixed, the total signal was radiated by the transmitting signal and received by the receiving antenna. In the receiving module the convolution of the received signal was made, for this purpose it was fed to an identical modulator, to which an identical chaotic binary sequence was fed.

In an experiment to determine noise immunity, two types of interference were used: sinusoidal interference, close in frequency to the transmitted microwave signal, and broadband interference matched by the spectrum with the transmitted signal (Fig. 15). Broadband interference was formed using the microwave phase manipulator

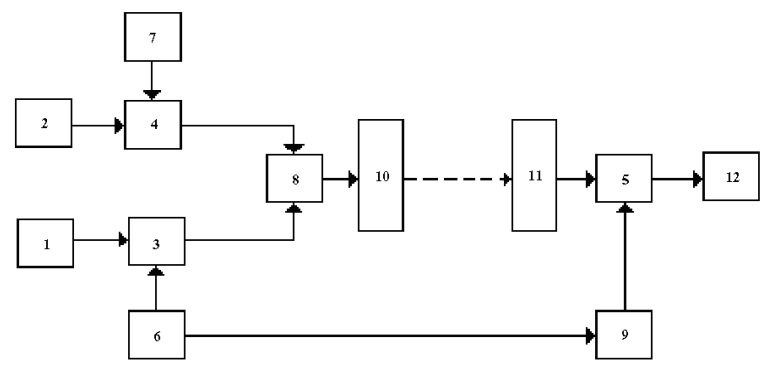

Fig. 15. Block diagram of an experiment to study noise immunity of a channel with a noise-like carrier. Microwave signal generators and interferers -1 and 2, respectively; phase modulators $\Phi M 1$, $\Phi M 2$ and $\Phi M 3$ - 3, 4 and 5; encoders 6 and 7; Microwave totalizer - 8; adjustable delay line - 9; transmitting and receiving microwave antennas - 10,11, spectrum analyzer -12.
FM2 (4). Control of FM2 (4) was carried out by a separate encoder (7) with the same clock frequency as the encoder (6) of the transmitter. The coding sequences of the two encoders (6) and (7) are different and uncorrelated in time. The experiment was performed with the synchronization of the coding sequences for the modems of the transmitter and receiver, which was provided by the use of an adjustable delay (9). The reverse coherent compression with respect to the frequency of the received signal was made by the modem (5) in the receiver circuit.

As a criterion for noise immunity, the coiled signal was exceeded at the output of the receiver (12) above the noise background, depending on the signal-to-noise ratio at the receiver input.

In Fig. 16 shows the results of measuring the signal-to-noise ratio $\left(S / S_{n}\right)$ at the output of the receiver depending on the ratio of interference

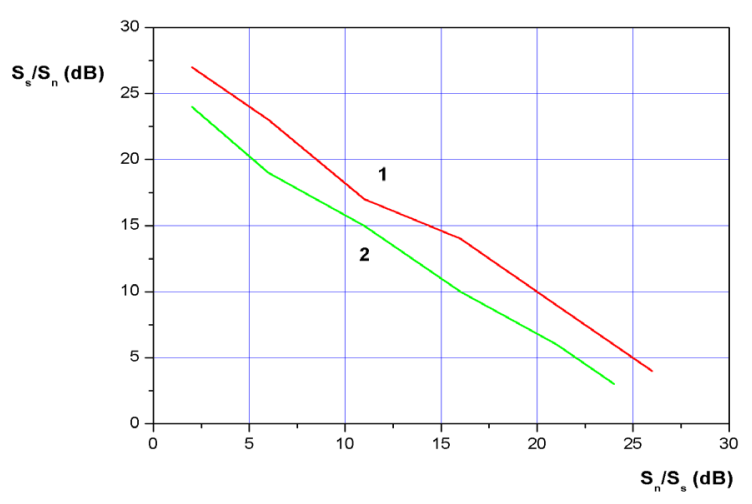

Fig. 16. The signal-to-interference ratio (Sc / Su) at the output of the receiver depending on the ratio of the interference levels and the signal at the receiver input (Sm / Sc) for two kinds of interference: narrowband (1) and broadband (2). 
levels and the signal at the receiver input $\left(S_{n} / S_{c}\right)$ for two kinds of interference: narrowband (1) and broadband (2). The maximum interference immunity for a radio channel with spreading is determined by the signal-to-noise ratio at the receiver input, at which signal restoration becomes impossible for a given averaging time.

Thus, the experimental results show that for both types of interference, the maximum noise immunity is $\sim 25 \mathrm{~dB}$. Under the influence of narrow-band interference, the spectrum of the total signal and interference at the receiver's input has the form of continuous in the band broadband noise corresponding to the received signal with spreading, above which the sinusoidal noise rises. The decoder in the receiver ensures convolution and extraction of a useful signal. Simultaneously there is a "blurring" of the power of narrow-band noise across the spectrum in the entire band, turning the noise into a noise pedestal over which the convoluted signal rises.

\section{CONCLUSION}

Practically all modern users of radar systems require an increase in the number and quality of information obtained from the observed space. Multifunctionality and efficiency of modern radar systems can be provided by the development and application of effective broadband technologies, unconventional digital algorithms and new adaptive applied solutions for the problem of processing signals and images in order to identify and recognize various classes of low-contrast objects.

In the present work, a noise radar model operating in the 8- $\mathrm{mm}$ band and having a frequency spectrum of the noise signal frequency up to $1 \mathrm{GHz}$ is developed, developed and studied on the basis of the noise generator. The layout of the model includes an LPD noise generator module based on a one-diode circuit operating in an 8 millimeter wavelength range with an integrated output power of $40 \mathrm{~mW}$ and a non-uniform spectral response of about $6 \mathrm{~dB}$. Receiving and transmitting modules include antennas, waveguide path elements: matching elements, decoupling, directional couplers, detector heads, attenuators. The computerized control unit provided program-algorithmic support of operating modes and double spectral processing of the signal.

An experimental study of the noise radar model in the laboratory demonstrated a sufficiently high accuracy of the radar range measurements in the entire measurement range for dual spectral processing of the signal, as well as a high resolving power over a range of $15 \mathrm{~cm}$ (with an effective bandwidth of $800-900 \mathrm{MHz})$.

The noise immunity of a radio engineering channel model based on a noise-like carrier was experimentally investigated. The maximum noise immunity for a radio channel with spreading was determined by the signal-to-noise ratio at the receiver input, at which signal restoration becomes impossible for a given averaging time. In the noise immunity experiment, two types of interference were used: sinusoidal interference, close in frequency to the transmitted signal, and broadband interference matched to the transmitted signal by the spectrum. The results of the experiments showed that for both types of interference, the maximum noise immunity is $\sim 25 \mathrm{~dB}$.

Thus, ultra-wideband radar technologies based on noise signals are characterized by good electromagnetic compatibility, can be effectively used in the compilation of a radar portrait of an object, as well as in monitoring, positioning and control systems operating under conditions of intense interferenc

\section{REFERENCES}

1. Immoreyev IYA. Ultra-wideband radars: new opportunities, unusual problems, system features. Bulletin of MSTU. Ser. Instrument making, 1998, 4: 25-56.

2. Bunkin BV, Reutov AP. Directions of development of radar systems. High technology, 2002, 4:8-12.

3. Kolesov VV, Potapov AA. The Information Technologies on Dynamic Chaos for Telecommunication, Radar and Navigation Systems. Electromagnetic Phenomena, 2005, 5(2(15)):89-104.

4. Potapov AA. The Textures, Fractal, Scaling Effects and Fractional Operators as a Basis of New Methods of Information Processing and Fractal Radio Systems Designing. Proc. SPIE, 
2009, 7374:73740E-1-73740E-14 (http://spie. org/x648.html?product_id=829032).

5. Verba BC, Dod VK, Trofimov AA, Chernyshev MI. Application of ultrashort pulses in radar systems of airborne patrol complexes. Materials I Int. Conf. "Ultra-wideband signals and ultrashort impulses of the radar, communications and acoustics". Suzdal, 2005. Moscow, Proceedings of the RNTORES them. A.S. Popov, 2005, no. 1.

6. Skosyrev VK, Osipov ML. Features and properties of short-pulse radar. Bulletin of Bauman MSTU, Specialist no. "Radioelectronics", 1999, №4.

7. Chapursky VV. Uncertainty functions of UWB multifrequency signals. Tr. Conf. "Ultra-wideband signals and ultrashort pulses in radar, communications and acoustics." Suzdal, 27-29 Sept. 2005, p. 21-25.

8. Bystrov RP, Dmitriev VT, Potapov AA, Sokolov AV. Questions of perspective radiolocation. Ed. A.V. Sokolov. Moscow, Radiotekhnika Publ., 2003, p. 20-48.

9. Kolesor VV, Fionov AS, Gorshenev VN. The modeling of the radioabsorbing medium on the basis of composite materials from the polyvinylchloride plastisols. RENSIT, 2014, 2(2):138-161.

10. Varakin LE. Communication systems with noise-like signals. Moscow, Radio i svyaz Publ., 1985.

11. Belyaev RV, Kalinin VI, Kolesov VV. Interference immunity of a spread spectrum communication system based on chaotic binary codes. J. of Communications Technology and Electronics, 2003, 48(10):1157-1185.

12. Belyaev RV, Kalinin VI, Kolesov VV. Formation of a noise-like carrier in spread spectrum communication systems. J. of Communications Technology and Electronics, 2001, 46(2):214-223.

13. Belyaev RV, Vorontsov GM, Kislov VYa, Kolesov VV, Krupenin SV, Popov AM, Ryabenkov VI. Complex Chaotic Discrete Signals in Telecommunications, Radar and Navigation Systems. J. of Communications Technology and Electronics, 2006, 51(9):1116-1128.

14. Grinev AY. (ed.) Broadband and ultra-wideband signals and systems. Moscow, Radiotekhnika Publ., 2009.

15. Solovyev NA, Slukin GP, Chapursky VV. Model of radio image of objects in the holographic radar systems of the planned analysis. J. of Communications Technology and Electronics, 2018, 63(6):619-624. DOI: 10.7868/ S0033849418060153.
16. Kalinin VI. Ultra-wideband radar with double spectral processing of noise signals. Radiotekhnika, 2005, 3:25-35.

17. Astanin LYu, Kostylev AA. Fundamentals of ultrawideband radar measurements. Moscow, Radio i svyaz Publ., 1989.

18. Radzievsky AT, Trifonov PA. Processing ultrawideband signals and interference. Moscow, Radio i svyaz Publ, 2009.

19. Chapursky VV, Sablin VN, Kalinin VI, Vasilyev IA. Wideband Random Noise Short Range Radar with Correlation Processing for Detection of Slow moving objects behind the Obstacles. Proc. of the 10th Intern. Conf. on Ground Penetrating Radar. Delft, Netherlands, 2004, p. 199-202.

20. Kalinin VI, Chapursky VV. The effectiveness of the dual spectral analysis. J. of Communications Technology and Electronics, 2006, 51(3):303-313.

21. Zalogin NN, Kalinkevich AA, Kirillin KA. Calculation of the signal-to-noise ratio for a radar station. J. of Communications Technology and Electronics, 1993, 38(2):278.

22. Kalinin VI, Chapursky VV, Cherepenin VA. Ultra-wideband noise radar with high spatial resolution. Tr. 3rd All-Russia. scientific and technical. Conf. "Radiolocation and radio communication". Moscow, 2009, p. 194-196.

23. Kislov VYa, Myasin EA, Zalogin NN. On nonlinear stochastization of self-oscillations in an electron-wave generator with delayed feedback. J. of Communications Technology and Electronics, 1980, 25(10):2160.

24. Kalyanov EV, Kalinin VI, V.Ya. Kislov VYa. Parametric excitation of complex and chaotic oscillations in a dynamical system with a resonator in a chain of delayed feedback. Journal of Communications Technology and Electronics, 2002, 47(8):984-997.

25. Kalyanov EV, Kalinin VI. Autostochastic system with parametric excitation. Technical Physics Letters, 2002, 28(13):46-50.

26. Myasin EA, Kotov VD. Broadband diode generators of millimeter wave noise. Radiotekhnike, 2005, 3:46-50.

27. Kalinin V, Panas A, Kolesov V, Lyubchenko V. Ultra wideband wireless communication on the base of noise technology. Proc. 16th Intern. conf. on microwaves, radar and wireless communications, 2006, Krakow, Poland, 2:615-618. 
APPENDIX NOISEOTRON OR MY LIFE IN THE 16th DEPARTMENT OF IRE RAS

\author{
Myasin E. A.
}

Kotelnikov Institute of Radioengineering and Electronics of RAS, Fryazino Branch, http://www.cplire.ru Fryazino 141190, Moscow Region, Russian Federation

eam168@ms.ire.rssi.ru

\section{Content}

1. Dofryazinsky Period IN THE LAB. 162 (250)

2. Fryazino PERIOd (252)

3. Noise Radar and Broadband Diode Noise Generators (253)

4. Dashing 90's (254)

5. Conclusion (255)

REFERENCES (255)

1. DOFRYAZINSKY PERIOD IN THE LAB. 162 I read not without interest this narrative on the theme "My Life in Science" by my colleague Mr. N.N. Zalogina. Of course, it seems to him that everything was exactly as he remembers or wants to remember. Will have to continue in the same spirit.

I will not correct the author's memories of events related to my person in terms of obtaining a broadband noise signal in the ring system of two TWTs, but I will still make some observations about some events in my being of development and the formation of "chaotic" subjects in the laboratory V.Ya. Kislova. Especially since I was his deputy from 1968 to 1980. Yes, indeed, I, Myasin EA, moved to work at IRE from the company PO Box 1598 in Sredmash a year after the end of MEPhI in order to become and have the opportunity to participate in international competitions abroad. It happened on October 2, 1962. I was not yet a master of sports, but I really wanted to be from childhood. The working atmosphere in the laboratory was so creative and interesting, and most importantly, it concerned completely unfamiliar technical fields (microwave electronics, technical electrodynamics, plasma theory, etc.) that I spent about a year mastering these new fields of knowledge. I have learned something from literature in MEPhI. Well, I did not forget the university course of mathematics and physics. He studied with pleasure and even with frenzy, because he did not want to be lagging behind. In the process of this "acclimatization" head. lab. I was attached to the work of various experimental groups so that I could learn with a new experimental technique for me. My young teacher, Erdeni Vladimirovich Bogdanov, was my mentor and teacher, a model of attitudes toward obtaining experimental results, over the next few years. Under his leadership, I, after all, remained on the installation of the first pulsed ruby laser in IRE, received from Z.S. Chernova, "underground" nickname "caretaker laser beacon," and became a co-author of my first publication in a private press on the heterodyning of laser radiation using photo TW'T ten-centimeter range.

Despite the lack of mutual understanding, which happened later with years of teamwork, I am grateful to V.Ya. Kislov, and because he took me to his laboratory and gave me an opportunity to comprehend the wisdom of a new science and technology for a year, and I will remember this for the rest of my life.

Probably, the laboratory staff first looked at me with suspicion, still "stranger", but I did not notice, they were all pretty intelligent people: both scientists and laboratory assistants.

At that time, the staff of the laboratory 162 was remarkably friendly: they celebrated the significant dates of the employees, celebrated holidays, went to demonstrations, and later even ate my hunting trophies-a wonderful wood-grouse noodles and two five and a half kilogram wood grouses.

I think that V.Ya. Kislov did not regret that he took me to work, like many other employees, whom I once was a "stranger" and who, thanks to the discovery of the noise, were able to defend their Ph.D. theses and not only them. Today, unfortunately, I can not ask V.Ya. Kislov this question, since he died after the tragic events that happened to him in the 90s. And if it were not for these events, he probably still had a lot to do, because he was truly devoted to science, he was talented in everything: in understanding the physics of phenomena, in 
developing theoretical models, in organizing work and in recruiting personnel. I was his first graduate student. He did not teach me anything, but I learned a lot from him!

It is appropriate to recall here the mentioned topic "Saber", which characterizes in many ways the character of V.Ya. Kislov, his style of work and decision-making. This was the first contractual work, in which the "severe" parameters of the broadband noise signal, necessary for the presentation of the commission for acceptance of work, were recorded. On this TK in the IRE before this, apparently, no one dared. In the Institute wall newspaper, who apparently knows about this TK anonymous wrote under the photograph of V.Ya.: "The Saber is hanging over you sharp, it's quite easy to cut yourself off!"

The co-executor of the work was the Kharkiv Physicotechnical Institute, in which a model of a noise generator was created on the basis of a self-made electrodynamic spiral-plasma system. I (according to the memoirs of NN Zalogin, a young specialist) went to Kharkov and meticulously carried out the acceptance of this part of the work. For which he paid, because he was late for the return train Kharkiv-Moscow due to a joyful feast in connection with the recognition that I had completed the work on time and in full. I had to get to Moscow in the trolley of a freight train, where I was allowed to load after meeting my "untried" with the station's head.

In our laboratory, such a generator was a Vesnianka TWT, $10 \mathrm{~kW}$ of the UHF of the decimeter range, which operated under continuous pumping. This TWT, in which instead of the usual Pierce gun was built a magnetron gun (as noisy), V.Ya. got it in "Titan", or, as they said then, in the Southwest. TW'T worked under continuous pumping, and I was responsible for working on this high-voltage stand. We have generated broadband noise in the entire TWT band, varying the pressure of the residual gases in its volume, but in the form of noise bands with a very poor degree of overlap. Here N.N. Zalogin is right, there was not enough reinforcement. The output power of the generator was, of course, not $10 \mathrm{~kW}$, but of the order of $5 \mathrm{~kW}$ exactly, and in continuous mode. It happened a little before my business trip to Kharkov, so I used to pick on them.

The work was successfully commissioned by the State Commission, in the "peak" to ill-wishers, but not without an emergency. While explaining the principles of Vesnyanka's work to members of the commission, I, as I had never done before, put my elbow in a metallized dressing gown on a metal frame that served as a fence and an electrical safety margin when working at the stand. The result was not slow to show itself. From the negative electrode of the rectifier connected to the electron gun of the TW'T (the lama collector was at the floor), there was a breakdown of the air gap of 20-30 centimeters on the fingers of my hand. There was a spark and a characteristic crackle. I rested my hand, but the members of the commission were frightened. It happened in 1964.

This passion for work did not pass for me in vain. In the same 1964 my team SK "Kuntsevo" became a bronze medalist of the USSR Championship on a hand ball, and me and a number of other team players were awarded the title of Master of the USSR sort of handball. During the presentation of the certificate, the coach invited me to become a professional athlete.

The conversation was like this. "How much do you get?" - "120 rubles." - "You will receive 150 rubles, be listed at the plant as an engineer and train four times a week. Have agreed? ". And I answered without hesitation: "No!", Thus signing the end of my sports career, not immediately, but, as it turned out, in the foreseeable future.

Nevertheless, training and competition continued in 1965, and in 1966, and so until 1974, although, in 1970, already in the team of the second division "Locomotive". I was away from work for training and competitions, taking leave at my own expense. At first these were absences within the Union, but in 1966 my team became the USSR Champion and got the right to play for the European Champions Cup, and it was necessary to go abroad. I needed a description from the Party Committee and the Trade Union Committee, and I received it, and traveled to many countries of Europe, thanks to the fact that Zarem Sergeevich Chernov supported me signing my positive characteristic for me, I do not know why I trusted. Of course, I could not fail him and never failed. In addition, I owe him and the fact that he became the head of the laboratory 168. And, of course, I always remember this. Unfortunately, he has not been with us for a long time. 
But between the fees and competitions I still worked. And, after the break with a special pleasure. Be that as it may, it was in 1966 that I was fortunate enough to deal with the TWT modes in the noise circuit, about which N.N. Zalogin.

\section{THE FRYAZINO PERIOD}

In 1970, I, like most employees of the lab. 162, went to work in the lab. 166 of the Fryazino part of the IRE, as mentioned above, and continued to work on the properties of various variants of noise noise and its possible introduction into various systems. At the same time, we had to interact with the staff of the lab. 168, engaged in the implementation of research results lab. 166 and the lab. 169, which will be discussed in more detail later.

After the award in 1980 of our work of the USSR State Prize, the leadership of the department in the person of Z.S. Chernova (through the parliamentarian NN Zalogin) invited me to participate in the competition for the post of head of the laboratory 168, which was temporarily performed by V.V. Evdokimov.

Unlike the choice of the professional sportsman's activity, not only money was put on the scales (400 rubles head of the lab, instead of 300 rubles), but, in general, my subject. I had to jump off the train that I had borne, which was gaining momentum. There was something to think about! But then I remembered (in general, I never forgot) that in the laboratory of 166 I worked in the position of the doctor of medical sciences. until 1977 (after defending his Ph.D. thesis in 1970). I did not address my boss with a request to transfer me to the position of senior researcher, moonlighting as an expert in the Supervisory Board of the VPTB, sometimes going to throw everything to ... .mater. And the decision was made: I agreed and on February 4, 1980 I became the head of the laboratory 168. And today I do not regret this at all! V.V. Evdokimov became my deputy. In tandem with him, we continue to work today.

So, formally, I left, but still stayed in this "noise" and, as I understand today, could not help but stay. But now I myself determined what to do when and to whom. The first four years were given to the reorganization of work in the laboratory, although in VNTL V.Ya. Kislov, I was doing something inessential. The situation was not simple, the topic was diametrically opposed to what was in the laboratory 166.

Before continuing on this narrative, it is necessary to make a small digression and note the contribution of the lab. 168 in the development of the direction associated with the generation of stochastic signals. About this, for some reason, I forgot to mention in my story NN. Zalogin.

Laboratory 168 was organized in the Fryazino part of the IRE Academy of Sciences of the USSR in January 1966 at the initiative of Academician ND. Devyatkov. The purpose of its creation was the development on the basis of ideas generated in the 16th Division, semi-industrial experimental samples of new electrovacuum microwave devices to accelerate the process of their introduction into industry. For this purpose, with the help of the Istok enterprise, the laboratory was equipped for two years with technological equipment that, in principle, allows the creation of sealed-off electrovacuum UHF devices in both metal-glass and metal-ceramic designs and conducts their experimental research. Highly qualified specialists of technologists were involved along with young specialists. The head of the laboratory was at this time I.M. The wretched.

The first step of laboratory 168 (1966-1968) was the creation on the basis of theoretical and experimental results obtained in the lab. 169 (head of the Laboratory of Physics and Mathematics, IF Kharchenko), sealed metal-glass samples of plasma noise generators of the decimeter range with an output power of the order of $1 \mathrm{~W}$ in continuous mode and a power spectral density (2-5 ) x10-3 W / $\mathrm{MHz}$ in the band 600-1400 MHz.

The next direction of the laboratory (1969-1974 gg.) Was the development and creation of a series of powerful sealed metal-ceramic samples of the noise generator together with employees of the lab. 166 (head of the laboratory Dr. V.V. Kislov). For the first time in the USSR, a one-cylinder continuous-mode noise generator with an output power of $2.4 \mathrm{~kW}$ in the $100-1400 \mathrm{MHz}$ band and a noise generator with an output power of $600-700 \mathrm{~W}$ in the $300-3500 \mathrm{MHz}$ band was created (Fig. 1). However, due to the lack at the time of effective small-size magnetic focusing systems, these developments were not introduced into the industry.

Nevertheless, already in 198? year, one copy of a one-balloon decimeter of the decimeter range was 
supplied for the research of the operation of the equipment in one of the enterprises of Moscow.

At the moment of my arrival in the lab. 168 in 1980 she led (since 1974) the study of physical processes in an orotron-generator with an open resonant electrodynamic system and longitudinal electron-wave interaction. Temporary Acting Head. the laboratory was Valery Vyacheslavovich Evdokimov. Actually, the management was carried out from the Moscow part of the IRE directly to Z.S. Chernov and Grigory Alexandrovich Bernashevsky. The experiments were conducted in a $3 \mathrm{~cm}$ wave band. Again had to learn! But about the orotron a separate conversation, and now about the return to "native penates".

\section{NOISE RADAR AND BROADBAND DIODE NOISE GENERATORS}

In 1984, a card for the development of a noise radar of $8 \mathrm{~mm}$ range came into 1 department. I do not remember why I was introduced to her. Probably, according to old memory. I went to V.Ya. To Kislov. I ask: "Will you do this work?" He answers: "No. I have so much to do about it! ". "Then I undertake, and you will also be the leader, but at first your employees will help in the $3 \mathrm{~cm}$ range, because no element base $8 \mathrm{~mm}$ range, not to mention the noise generators that need to be created, not available. Do you agree? "-" Yes. " Thus began my penetration into the millimeter wavelength range and a return to the generation of stochastic oscillations already in generators on avalanche-passing diodes. Thus, the beginning of the mastering of the millimeter wave band (MMDV) by broadband diode noise generators in the IREE of the Academy of Sciences of the USSR belongs to 1984 and is associated with the formulation of works on noise radar in the $8 \mathrm{~mm}$ wave band [1], and the "man from the Troika" about which NN mentioned. Zagolin, was Yu. Yakimchuk.

To begin such studies (without having any element base of the $8 \mathrm{~mm}$ range), a large amount of experience accumulated by this time in the IRE of the USSR Academy of Sciences on the phenomenon of the transition of oscillators of harmonic oscillations to the mode of generating broadband noise has allowed. These studies were carried out not only in electron-wave systems with delayed feedback (AOS) [2-5], but also in semiconductor diode generators of the centimeter wave band made by N.N. Zalogin and R.V. Belyaev [6]. This phenomenon, later called the "nonlinear stochastization of oscillations in dynamical systems," was discovered in the Institute of Physics and Engineering of the USSR Academy of Sciences in 1966, for the first time both in our country and abroad, and then studied in detail in electron-wave systems with (ZOS ) [2]. Unfortunately, as N.N. Zagolin, because of the important applied importance of these studies in the microwave range, their main results were published much later [2-5], when in the scientific literature a real boom began to study this fundamental property of nonlinear dynamical systems of various nature. Investigations carried out at the Institute of Physics and Technology of the Academy of Sciences of the USSR [2-5] led to the creation of a special class of generators of stochastic (noise) oscillations - "noise wheels", the features of which were considered in [7].

The basis for the construction of experimental models of diode GS was put the necessary signs of stochastization of oscillations in electron-wave systems with a delay, which made it possible to begin the development of the $8 \mathrm{~mm}$ range from the experiment and only after some time to consider the theoretical model of such a generator [8]. A comparative analysis of the characteristics of the noise signal of generators using Gunn diodes and avalanche-passing diodes (PDD) in the centimeter wavelength range, performed by the authors of Ref. 6, determined the choice of the active element for the noise generator in favor of the latter. LAPs were also used in noise generators (GSLPDs) of the millimeter wave band.

Great contribution to the development of this direction in the lab. 168 was brought in by the engineer of the laboratory, and now by the doctor of science, Viktor Dmitrievich Kotov, whom I managed to persuade to switch from "orotron" subjects to "solid".

Of course, the agreement on the creation of the $8 \mathrm{~mm}$ wide SHRLS layout was successfully implemented. GSLPD was created. Experiments were conducted to investigate the work of this locator, in which a large share of participation was Valery Ivanovich Kalinin. But the most important thing is that at the expense of this contract 168 firsttime instruments and a 8-mm HF path appeared in laboratory 168. Further for a number of years 
work was carried out to improve the design of this GSLPD, its characteristics, the process of transition to noise generation mode and the possibility of creating SHRLS based on it for various applications were studied. It was shown the possibility of overlapping only $8 \mathrm{~mm}$ of the range 4 - 5 GSLLPD with a spectrum width of $800 \mathrm{MHz} . .1000 \mathrm{MHz}$. All this was done according to the contracts with the Customers, the financial means from which provided the possibility to continue equipping the laboratory with equipment of this range, which was very useful for moving the orotron in the $8 \mathrm{~mm}$ range. Although the work under the Agreements was conducted according to a closed plan, after all, with the permission of the Customer, it was possible to publish something in the open press, for the first time in 1990 [9]. Then, the need for noise generators of $3 \mathrm{~mm}$ range appeared in the Customers. This range was also mastered in the pre-perestroika period. GGLPDs were created with a $3 \mathrm{~mm}$ range with a spectrum width of up to $8 \mathrm{GHz}$, operating at the 2nd harmonic generation of the $8 \mathrm{~mm}$ range LDP produced in Nalchik. The theory of this GSLPD was considered in [10]. A young specialist, and later a graduate student, Yury Veniaminovich Andreev, made a big contribution to the theoretical description of the process of transition of the GSLPD to the mode of noise generation. And in this case, the money earned made it possible to equip the laboratory with equipment and a waveguide channel of $3 \mathrm{~mm}$ range. So $3 \mathrm{~mm}$ panoramic meter VSWR, which m.ns. lab. 168 Boris Andreevich Belyavsky brought after the alteration of a lower-frequency device from Vilnius, and works today.

\section{DASHING 90's}

In the dashing 1990s, reformist liberals (and, in fact, the second echelon of the party nomenclature) seized power in the USSR, disrupted the Great Soviet Empire, and overnight liberated the verbiage of the liberal media that muddied the Soviet Union and brought down the financing of science, military industry, education, medicine and ... Millions of Soviet people were actually unemployed and did not receive wages for months. So employees of the cathode department - one of the main departments of the FGU NPP "Istok" - in the morning before the work went to put the tents on the market, and in the evening to clean them. Speculators, bandits and thieves, who accumulated millions on trading bases, in stores, in state bodies of material values management during Gorbachev's perestroika period, were able to only partially legalize them. They welcomed the new power, becoming masters of real estate, factories, steamships, etc. Millions of ordinary people, in order to survive, became "shuttle traders", and the whole country turned into a consumer goods market. To feed their families, some of the qualified specialists left the country, and a part that did not want to leave their homeland was forced either to become street vendors and quit their favorite work or to survive in beggarly conditions, trying to save a business that has invested more than one year of life, adapting to existing conditions. Of course, there were some who followed the veiled advice of the devil: "Enrich yourself!" By Mr. Yeltsin. Or they were expected to be corrected in accordance with the question of $\mathrm{Mr}$. Berezovsky, incidentally, corresponding member of the Academy of Sciences of the USSR: "If smart, why poor?". This public imposed on the citizens of the country, in the recent past, Soviet people, instead of the 10 Christian commandments (the code of honor of the builders of communism) their mentality of the leadership of the loan interest and successfully continues to do this through their media today.

In these troubled years I had to find ways to save the business, to find at least some kind of financial assistance to my employees and myself. Saved the noise theme. It was necessary, along with

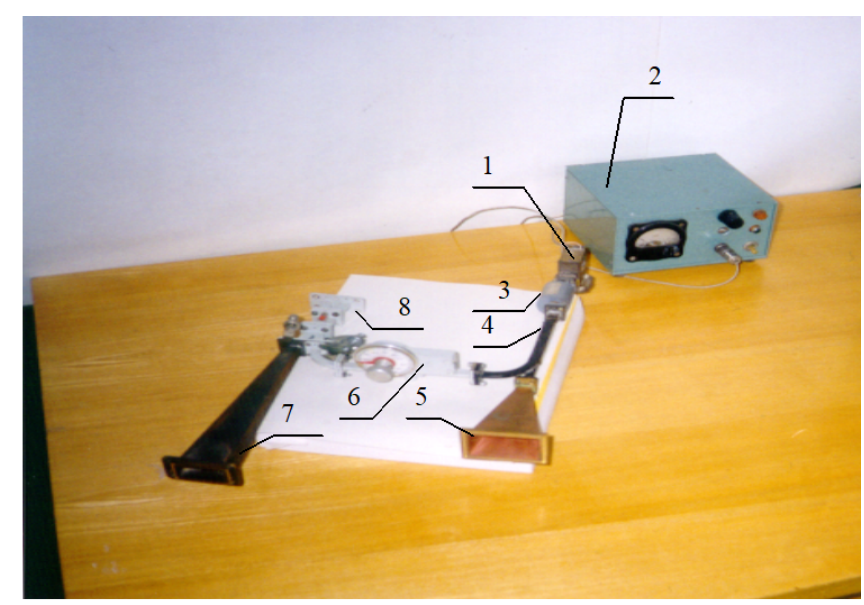

Fig. 1. Receiving-transmitting block of SHRLS.

1 - noise generator (GSH) of $8 \mathrm{~mm}$ wave band, 2 - power supply,

3 - ferrite isolation valve, 4 - directional reference signal coupler, 5 - emitting horn, 6 - attenuator, 7 - receiving horn, 8 - adder of received and reference signals. 
academic themes, to deal with EHF-therapy (thanks to ND Devyatkov and the successor of his case to Oleg Vladimirovich Betsky). It was decided to use relatively narrow-band GGLPAs that generate a noise signal near the known wavelengths of $7.1 \mathrm{~mm}$, $5.6 \mathrm{~mm}, 4.9 \mathrm{~mm}$ as sources of radiation in devices for EHF therapy $[11,12]$. It was organized by LLC "SHLEM" (founders EA Maisin and VD Kotov), which produced and successfully sold devices with the same name. In this work, most of the technical staff of the laboratory were involved. Naturally, the results of clinical trials of these devices were published, not only in our country, but also abroad [13]. In the end, in 2002 LLC ordered "a long life", as I realized that it's already hard to sit alone on several chairs and you need to make a choice.

\section{CONCLUSION}

To the noise theme already within the framework of academic programs of the lab. 168 has addressed in the future [14]. In 2002, a new method for analogto-digital signal processing in SHRLS was proposed [15], implemented experimentally on a laboratory model of SHRLS [16, 17]. At the same time, the results of our experimental studies on GSLP were published [18]. Currently, these GSLPDs are used in experimental radio-vision systems at FIDE named after. V.A. Kotel'nikov Academy of Sciences [19].

\section{REFERENCES}

1. Volzhin VN, Yakimchuk SE, Kislov VY, Kalinin VI, Measin EA, etc. The method of radar. AS No. 792183, BL. №48, December 1980

2. Measin EA, Kislov VY, BogdanovEV. Method for generation of electromagnetic noise oscillations: A.C. N1125735, published on 23.11.84. BL N43 with priority from June 22, 677

3. Kislov VY,Zalogin NN, Myasin EA. Investigation of stochastic autooscillatory processes in selfexcited oscillators with delay. Radio Engineering and Electronics, 1979, 24 (6): 1118.

4. Kislov VY, Myasin EA, Zalogin NN. On nonlinear stochastization of auto-oscillations in an electron-wave generator with delayed feedback. Radio Engineering and Electronics, 1980, 25 (10): 2160.

5. Kislov VY. Theoretical analysis of noiselike oscillations in electron-wave systems and self-excited oscillators with delay and strong nonlinearity. Radio Engineering and Electronics, 1980, 25 (8): 1683.

6. Belyaev RV, Zhernovenkov AS, Zalogin NN, Melnikov AI. Experimental study of the excitation of noise oscillations in the GLDD. Radio engineering and electronics, 1996, 41 (12): 1484-1489.

7. Anisimova SE, Vorontsov GM, Zalogin NN, Kislov VY, Myasin EA. Chromotron. Radio Engineering, 2000, 2: 19-25.

8. Andreev SE, Myasin EA. Investigation of the dynamics of the model of an odd-diode generator on the LPD. Radio Engineering and Electronics, 1989, 33 (11): 2358.

9. Myasin YeA, Kotov VD, AndreevYuV. Ka-Band Lardge Bandwidth Noise Signal Source. Proc. 15th Intern. Conf. on Infrared and Millimeter Waves. Dec. 10-14, 1990, Orlando, Florida, USA Conf.Digest, pp. 631-633.

10. Andreev SE, Myasin EA, Kotov VD. On the problem of generation of the second harmonic in the model of an autostochastic oscillator on an avalanche-spill diode. Radio engineering and electronics, 1991, 36 (2): 426-428.

11. Myasin EA, Kotov VD, Andreev SE. A generator with a nondeterministic signal of the MM range for biomedical research. Thesis VII. Vses. sem. "Application of low-intensity EHF radiation in biology and medicine". 13-15 Nov. 1989, Moscow, p. 134.

12. The meat of EA, Kotov VD. The device for EHF therapy "Helmet-1." Proceedings of Int. simp. "Millimeter waves of nonthermal intensity in medicine". Part 3. Moscow, 1991, p.739-734.

13. The meat of EA, Kotov VD. Application of EHF-therapy for hypertensive crises. Tr. Int. Conf. on hypertension, Tokyo, 1999, p. 108.

14. Myasin EA, Black LF. etc. Project 2.4, Study of the propagation of millimeter wave broadband signals in the surface layer of the atmosphere and determination on this basis of the limitations imposed on the extremely achievable characteristics of locating systems with noise and broadband signals. Sat. reports on the scientific projects of the Russian Ministry of Science and Technology "Physics of Microwaves". N. Novgorod, 2001, p.77-81. 
15. The meat of EA, Kotov VD. The noise radar. Certificate of utility model number 25797. Priority from 22.04.2002.

16. Massine EA, Ilyin AYU, Kotov VD, Chmil AI. Noise $8 \mathrm{~mm}$ radar wave band with spectral and digital signal processing. Tr. 4-th Intl. Conf. "Digital signal processing and its applications". Moscow, 2002, p. 210-213.

17. Massine EA, Kotov VD, Ilin AI, Chmil AI. Noise radar with analog and digital signal spectral processing. Radio Engineering, 2005, 3: 36-40.

18. The meat of EA, Kotov VD. Broadband diode generators of millimeter wave noise. Radio Engineering, 2005, 3: 46-50.

19. Materials of the reports of the FIDE RAS. Report lab. 176. 